The Egyptian Journal of Hospital Medicine (January 2018) Vol. 70 (4), Page 638-643

\title{
Lumbar Puncture: Indications, Complications, Technique and CSF Analysis in Pediatric Patients
}

\author{
Mahdi Hassan Alsuwailem, Ahmed Hassan Alkhatem, Abdullah Ahmed Alshabib, \\ Mohammed Hussain Aldraisi, Abdullah Khalid Al Jughiman, Mohammed Hussain Al-Turaifi, \\ Samirah Munther Al-Ali, Ali Hussain Al Nujaydi \\ King Faisal University \\ Corresponding Author: Mahdi Hassan Alsuwailem - MHS_1992@HOTMAIL.COM - 0561893332
}

\begin{abstract}
Introduction: The subarachnoid space is filled with cerebrospinal fluid, which circulates through the entire central nervous system aiding in providing nutrition, fighting microbes, and acts as a buffer. Additionally, the cerebrospinal fluid also helps in diagnosis of various pathologies affecting the central nervous system ranging from infection, trauma, and tumor in the pediatric population with the help of lumbar puncture. Methodology: We conducted this review using a comprehensive search of MEDLINE, PubMed, and EMBASE, January 2001, through February 2017. The following search terms were used: lumbar puncture, pediatric indication for lumbar puncture, contraindications to lumbar puncture, technique procedure, CSF analysis. Aim: In this review, we aim to study the indications, contraindication, and complications of lumbar puncture. We will also study the correct technique, as well as the analysis of cerebrospinal fluid. Conclusion: The lumbar puncture procedure is highly effective in diagnostics as well as therapeutically, however it can be fatal if gone wrong. There are several complications which can result from performing the procedure incorrectly, as well as some underlying pathologies can make it contraindicated.
\end{abstract} Keywords: pediatric indication for lumbar puncture, contraindications to lumbar puncture, technique procedure, CSF analysis.

\section{INTRODUCTION}

The subarachnoid space is filled by the cerebrospinal fluid (CSF). An adult produces around $500 \mathrm{~mL}$ of CSF every day, at a frequency of about $0.5 \mathrm{~mL} / \mathrm{min}$. In children of 4-13 years of age, the static CSF volume varies between 65 to $150 \mathrm{~mL}$. It is completely replenished every 4-6 hours. The volume extracted by a routine lumbar puncture (LP) is about $3-5 \mathrm{~mL}$, and is replaced in $<1$ hour $^{[1]}$. LP is a vital diagnostic and therapeutic tool. It is performed in order to obtain a sample of CSF. In children, the most common purpose for a LP is to diagnose an infection within the central nervous system (CNS) such as meningitis or meningoencephalitis.

Lumbar puncture is also essential for the diagnosis of various other non-infectious diseases in children including inflammatory disease like transverse myelitis, hematological-oncological conditions in case of CNS leukemia or lymphoma, as well as when subarachnoid hemorrhage is suspected, or in case of other metabolic disease. Besides, LP is necessary to confirm CSF opening pressure which helps to diagnose idiopathic intracranial hypertension. Less frequently, LP can aid for therapeutic reasons to administer chemotherapeutic or antimicrobial agents straight into the CSF.

\section{METHODOLOGY}

\section{-Data Sources and Search terms}

We conducted this review using a comprehensive search of MEDLINE, PubMed, and EMBASE, January 2001, through February 2017. The following search terms were used: lumbar puncture, pediatric indication for lumbar puncture, contraindications to lumbar puncture, technique procedure, CSF analysis

-Data Extraction

Two reviewers have independently reviewed the studies, abstracted data, and disagreements were resolved by consensus. Studies were evaluated for quality and a review protocol was followed throughout.

\section{Cerebrospinal fluid (CSF)}

CSF is a dynamic, metabolically active body fluid that does numerous important functions. It offers buoyancy to the brain, decreasing functional brain weight by around 75\%; and also aids to buffer nervous tissues from trauma. Since there are no lymphatics inside the central nervous system (CNS), CSF provides an essential fluid medium for chemicals and nutrients to sustain intercellular spaces, and works as a reservoir for neural metabolites to be given back to the venous 
circulation. Acute contraction in CSF volume can protect intracranial perfusion and preserve an adequate cerebral perfusion pressure in spite of conditions increasing intracranial pressure. CSF has antibacterial properties that prevent bacterial growth and proliferation ${ }^{[2]}$.

About $70 \%$ of CSF is made in the choroid plexuses of ventricles in brain, a microvillus structure lined by a single layer of epithelial cells joined together by tight junctions forming the bloodbrain barrier. In contrast with the vascularity of other organs, brain capillary endothelium does not have fenestrations or pinocytotic vesicles for transport. Furthermore, there are usually different ion channels and receptors on the extracranial cell surface unlike that on the intracerebral membrane neighboring brain parenchyma enabling cellular transport ${ }^{[2]}$.

CSF is ultra-filtrate of the plasma. CSF normally contains $99 \%$ water, and thus, looks clear and colorless. It contains electrolytes such as glucose, and proteins, enzymes, antibacterial factors, and a few white blood cells (WBCs); the concentration varies with age ${ }^{[3]}$.

The CSF circulation dynamic is moved by unidirectional secretory pressure produced at the choroid plexus. Values for the upper limit of normal CSF pressure are from 70 and $180 \mathrm{~mm} \mathrm{H}_{2} \mathrm{O}$. In a recent study on 500 children, CSF opening pressures was found to have the upper limit $\left(90^{\text {th }}\right.$ percentile) to be $280 \mathrm{~mm} \mathrm{H}_{2} \mathrm{O}^{[4]}$.

This continuous forward flow of newly derived CSF refills the subarachnoid circuit with fresh fluid and eliminates waste products. Magnetic resonance imaging has revealed that CSF movement through the system is pulsatile, helped by choroid plexus pulsations and by the motion of the cilia of ependymal cell ${ }^{[5]}$.

CSF made in the lateral ventricles streams via the intraventricular foramina of Monro, into the third ventricle, then from the cerebral aqueduct into the fourth ventricle, then through the foramens of Luschka and Magendie in the subarachnoid space. After leaving the ventricular system, most of the CSF goes into the subarachnoid cisterns at the base of the brain, while $20 \%$ flows reaches of the spinal subarachnoid space, which ranges caudally to the second sacral vertebra. The CSF that reaches the lumbar cistern is the sampled by a $\mathrm{LP}^{[3]}$.

\section{Indications and Contraindications}

Lumbar puncture must only be performed once a thorough neurologic examination is done. The most likely pediatric indication for CSF analysis is for diagnosis of meningitis. CSF analysis is the only fully reliable technique to the diagnosis. It is, therefore, routinely performed as part of a sepsis evaluation for febrile infants less than 8 weeks of age. Moreover, LP is performed in older children if the clinical presentation proposes risk for meningitis which includes ill/toxic appearance, headache with nuchal rigidity, focal seizure, altered mental status, and status epilepticus ${ }^{[6]}$.

Strong recommendation for sampling CSF to rule out meningitis must occur in any condition possibly associated with bacteremia. Other indications for CSF analysis comprise diagnosing Guillain-Barré syndrome and other demyelinating diseases, as well as for diagnosing and treating pseudotumor cerebri. Lastly, an LP assessment for the presence of blood in the CSF may be directed when subarachnoid hemorrhage is assumed while a computed tomography (CT) scan of the head is not diagnostic. When indicated, appropriate CT imaging should take place before LP to clarify safety of the procedure and rule out contraindications ${ }^{[6]}$.

LP is contraindicated in patients with suspicion for intracranial mass, or other indication pointing to increased intracranial pressure; hemodynamic instability like respiratory compromise; status epilepticus; any underlying spinal cord anatomic abnormalities, coagulopathy, or spinal or epidural abscess; or if there is an infection of the superimposing lumbar site such as cellulitis or skin or subcutaneous abscess. When indicated, appropriate CT imaging should precede LP to clarify if it is safe to perform ${ }^{[7]}$.

\section{Lumbar Puncture Technique}

Position and Landmark

LP is accomplished by positioning the child in the lateral decubitus position, fully flexed at the waist and neck in order to approximate knees to chin, while maintaining attention to tolerability of ventilation, that some endorse continuous pulse oximetry to confirm adequate ventilation through the procedure. Otherwise, for older children, a seated position can be used, with legs dangling over the examination table $\mathrm{e}^{[7]}$.

Either position should produce maximum spinal flexion to fully spread open the spaces 
between the lumbar spinous processes to enable the needle passage. To prevent lateral deviation of the needle into the spinous process or spinal nerve root in lateral decubitus position, the vertical plane of the patient's back should be positioned at right angle to the horizontal plane of the examination table. Deviance from this position will rotate the spinal column vertebrae, making needle insertion difficult ${ }^{[8]}$.

The significant landmark for deciding LP needle insertion site is established by palpating the lateral aspect of the superior iliac crest. Once the landmark is recognized, the supracristal line (which is a horizontal line between the two superior iliac crests) intersects the midline of the spinal column at level L3-L4 interspace. Using sterile technique, sterile gloves, and the skin overlying the lower lumbar spine is cleaned with povidone-iodine scrub in spreading concentric circles out all through the superior iliac crest $^{[9]}$.

\section{Pain Prophylaxis}

Some physicians advice local anesthesia with lidocaine infiltration before performing LP for infants. Evidence from trials in neonates proves that both infiltrative and topical anesthesia can decrease pain accompanying $\mathrm{LP}^{[\mathbf{1 0 ]}}$.

One can help ease pain in infants with administering oral sucrose anesthesia before LP. Topical application of EMLA cream (lidocaine and prilocaine) or lidocaine infiltration through $\mathrm{CO}_{2}$ jet atomizer can deliver painless topical anesthesia and diminish skin/subcutaneous tissue discomfort accompanying needle insertion, however does not penetrate deep enough to remove the need for subcutaneous lidocaine in the interspinous space. EMLA cream topical anesthetic needs 30-60-min application time to work, while lidocaine jet infiltration induces fundamentally immediate anesthesia. Older children can get a mild, shortacting sedative such as oral or intravenous midazolam to lessen anxiety and increase compliance ${ }^{[11]}$.

For older children, "long track" method of preLP lidocaine infiltration is preferred, where a $27-$ gauge, 1.5-inch-long needle injects anesthesia along the entire interspinous tract covering from skin to subarachnoid space before LP needle insertion. This confirms satisfactory anesthesia along the whole course that the LP needle will afterward navigate. The puncta created by inserting this smaller-gauge needle lacking resistance through the interspinous space aids in an accurate landmark for following spinal needle insertion ${ }^{[12]}$.

\section{Needle Insertion}

A 22-gauge spinal needle is favored in all pediatric ages; it will make a comparatively smaller rent in the dura unlike the larger-bore needles, and reduce the risk of consequent CSF leakage. Only the spinal needle length differs: the 1.5-inch length is used in ages less than 3 years, while the 3 -inch length is used for older children ${ }^{[13]}$.

The subarachnoid space is penetrated caudally after the termination of the spinal cord (which is at level of L1). The L3-L4 interspinous space is most suitable to enter, as the spinal cord endsabove this landmark. The level of the needle must be oriented in the sagittal plane, in order to spread rather than cut the fibers in the dural sac, as they run parallel to the spinal axis. The spinal needle is introduced into this interspace and gradually pushed perpendicular to the vertical plane of the patient's back. If correctly positioned, the needle must pass without bony resistance in this order through the skin, subcutaneous tissue, supraspinous ligament, interspinous ligaments, ligamentum flavum, epidural space, dura, and lastly, penetrate the subarachnoid space between the nerve roots ${ }^{[14]}$.

The spinal needle must be advanced cautiously, a few millimeters every additional intrusion, in a perpendicular plane to patient's back. Unlike an adult LP, the needle course must not be angulated, especially in younger-aged patients since pediatric spinous processes are comparatively flatter unlike adults. If bony resistance is experienced, the needle must be somewhat withdrawn back and redirected forward, adjusting for any angulation off the perpendicular; moreover ensuring the patient is fully flexed to allow the widest gap possible between two spinous processes ${ }^{[15]}$.

A "pop" tactile sensation can occasionally be sensed when the needle penetrates the ligamentum flavum and dura arachnoid, representing the subarachnoid space is entered. This sensation is often subtle, or even absent, in young patients since the ligament is quite thin ${ }^{[14]}$.

To prevent a traumatic LP, it is essential not to "overshoot" needle because an extensive venous plexus lining the ventral wall of the subarachnoid space can be perforated and result in hemorrhage ${ }^{[13]}$. 


\section{Image Guided Lumbar Puncture}

Conventionally, when an unguided lumbar puncture misses the mark, a fluoroscopically guided lumbar puncture is appreciated. Even though fluoroscopically guided lumbar punctures have been shown to reduce the rate of traumatic taps against unguided lumbar punctures, there are numerous reasons why fluoroscopic guidance is less efficient, especially in children. First, children are bare to ionizing radiation ${ }^{[8]}$. Even though the consequences have yet to be sufficiently studied, careful clinical practice is to reduce radiation exposure whenever possible. Second, fluoroscopically guided lumbar punctures are habitually performed in the prone position, which declines spinal flexion and often necessitates endotracheal intubation as well as general anesthesia for airway control. Third, fluoroscopically guided lumbar punctures cannot authorize intrathecal injection of medication at the time of injection without the use of contrast material. Lastly, fluoroscopy does not permit visualization of the thecal space, so occlusive thecal hematoma would be invisible ${ }^{[16]}$.

\section{Complications}

Post-Dural Puncture Headache (PDPH)

Most complications are negligible and temporary, including confined bruising, back pain, headache. They more common in adults, and usually resolves instinctively. Headache pain following a lumbar puncture was first noted in 1898. It was hypothesized that the reason of the spinal headache was leakage of CSF through the dura puncture site. PDPH is caused by the continuous loss of CSF through the dura hole shaped by the LP needle. The pain of PDPH after the loss of CSF by the dura puncture site is due to the resultant hypovolemia, compression on pain sensitive structures, and resulting venous dilation. Recumbent positioning allows CSF to flow into the skull, which declines the pressure difference across the dura along with the amount of CSF leakage to lesser pressure space. Recumbence position makes pressure to return to closer to equilibrium state and headache pain subsides $^{[17]}$.

The most significant factor in treatment of the PDPH is to avoid its occurrence. Using smallestgauge needle possible and technique of insertion can help avoid PDPH. Most of PDPHs resolve with minimal intervention. Since PDPH is self-limiting, conservative management is primarily indicated. Supportive treatment for PDPH and its accompanying symptoms include bed rest, analgesics, hydration, corticosteroids, and antiemetic medications. Moderate to severe headaches linked with nausea, vertigo, blurry vision, and back pain that do not decrease over a few days with conservative management may necessitate further interventions. In case of severe and persistent headaches, injecting saline into the epidural space may be therapeutic. Such mechanisms lead to greater than before intracranial pressure, nevertheless relief is temporary ${ }^{[18]}$.

\section{Herniation}

The most serious difficulty resultant an LP is post-procedural cerebral herniation. This is attributed to rapid CSF removal on top of underlying increased intracranial pressure (ICP). In such situations, CSF withdrawal via LP permits the elevated pressure compartment cephalad to move along the pressure gradient, with subsequently trans located CNS tissue. This is not likely with states of uniformly raised ICP within the entire CNS compartment such as, pseudotumor cerebri where there is no pressure gradient making LP safe to perform. Without meningitis, herniation that ends as neurologic deterioration or even death following an LP is uncommon; however, herniation can be possibly lethal in some patients with underlying bacterial meningitis and clinical evidence of elevated intracranial pressure. Herniation can be the direct cause of death in around $30 \%$ of such children ${ }^{[19]}$.

It is tough to establish direct cause and effect in some reported cases linking LP with following cerebral herniation, since many of these patients had an underlying intracranial pathology for example, bacterial meningitis with cerebral edema, which could have provoked the herniation process even without the effect of an LP. However, it is practical to perform cranial CT imaging to evaluate any such abnormalities before performing an LP. If there is any apprehension for elevated intracranial pressure, delaying this procedure until stability is recommended ${ }^{[9]}$.

\section{Traumatic Lumbar Puncture}

The most frequent complication while performing an LP is a traumatic tap. The incidence of traumatic LP is almost $20 \%$ for all children enduring the procedure, and can be as high as 33\% in neonates. The incidence of traumatic LP varies contrariwise with the age of the patient and the 
experience level of the performing physician. LP success rate has been shown to be two times higher in young infants who got pre-LP local anesthesia. A prospective study of 1459 children receiving LP exhibited that procedures done without local anesthetic were more probable to be traumatic or unsuccessful against those performed with local anesthesia ${ }^{[20]}$.

Traumatic LP is frequently the consequence of inserting the needle too far and penetrating the vascular plexus lining the anterior epidural space of the vertebral column. When peripheral blood elements pollute the subarachnoid compartment, it can make vague CSF analysis ${ }^{[21]}$.

\section{CSF Analysis}

Once indicated, CSF opening pressure measurement should be performed before withdrawing the CSF sample. A manometer is fixed to a three-way stopcock, which is set into the hub of the spinal needle, and CSF flows through the cylinder until attaining equilibrium. Accurate measurement of ICP is achieved while the patient is relaxed; lying in lateral decubitus position, with neck in neutral position and legs entirely extended provided the patient is able to comply with repositioning $^{[22]}$.

At least $3-5 \mathrm{~mL}$ of CSF must be routinely collected for analysis. The CSF sample must be transported to the laboratory by medical personnel itself. Processing must be accelerated, as neutrophil counts can reduce by up to $50 \%$ within 2 hours of collection $^{[23]}$.

It is imperative to routinely illustrate five indices of CSF composition:

1) Cellularity showing total $\mathrm{WBC}$ and red blood cell (RBC) counts

2) Chemistry (glucose and total protein concentrations)

3) Absolute neutrophil count (ANC)

4) Gram-stained smear, and

5) Bacterial culture

Total WBC and RBC counts are counted by placing a drop of unspun CSF on a Fuchs-Rosenthal counting chamber following microscopic analysis. A part of CSF is centrifuged, and a Wright-stained smear of the sediment is studied to provide a WBC differential count. A normal CSF must have an absolute neutrophil count $<1 / \mathrm{mm}^{3}$. A Gram-stained smear of the spun sediment must be inspected for the presence of bacteria and its products. Glucose and total protein concentrations must be counted. CSF is always cultured for presence of bacteria ${ }^{[24]}$.

At times, it is essential to perform ancillary CSF microbiologic testing. Polymerase chain reaction (PCR) is preferably suited for detecting fastidious organisms that may be otherwise difficult or nearly impossible to culture (such as, enteroviruses, herpes simplex virus, Borrelia burgdorferi, Epstein-Barr virus) has been particularly useful in the diagnosis of DNA and RNA viral meningitis ${ }^{[25]}$. Numerous viruses can cause CNS disease, comprising meningoencephalitis and post-infectious encephalomyelitis; even though the CSF profile is abnormal in majority (> 90\%) of cases, routine CSF studies often cause identification of a specific causative agent. PCR testing demonstrates high sensitivity and specificity for various infections of the CNS, and can be performed with a comparatively small volume of CSF. It is low-cost and rapidly finished, with results typically available within $24 \mathrm{~h}$. This permits the prompt introduction of empirical therapy when a patient presents with manifestations indicating suspected meningitis or encephalitis, without possibly compromising definitive diagnostic tests. In cases of HSV encephalitis, virus is rarely cultured from CSF in $<5 \%$ of cases. Nevertheless, in most cases, HSVspecific DNA can be distinguished by PCR ${ }^{[26]}$.

\section{CSF contaminated with blood}

The CSF becomes visibly opalescent when there are 200-500 $\mathrm{WBCs} / \mathrm{mm}^{3}$ present. Xanthochromia describes a yellowish discoloration of the sample when centrifuged. The pigments that add to xanthochromia are owing to the breakdown of RBCs into oxyhemoglobin, bilirubin, and methemoglobin ${ }^{[27]}$.

In case of traumatic LP, the initial CSF is more blood-tinged as the flushing efflux of sample. While in case of subarachnoid hemorrhage, the RBC count should remain steady between collection tubes since blood is evenly mixed throughout the $\mathrm{CSF}^{[28]}$.

\section{CONCLUSION}

CSF is an important body fluid, which can become infected and lead to significant morbidity and even death. Physicians are frequently called upon to perform an LP in children to rule out serious and even potentially life-threatening conditions. A successful procedure is enhanced by a thorough understanding of anatomy, the proper technique for 
performing LP, and normal CSF composition and alterations that occur with a variety of disease states.

The study was done after approval of ethical board of King Faisal university.

\section{REFERENCES}

1. Levin E, Muravchick S, Gold MI(1981): Density of normal human cerebrospinal fluid and tetracaine solutions. Anesth Analg., 60: 814-817.

2. Whedon JM, Glassey D(2009): Cerebrospinal fluid stasis and its clinical significance. Altern Ther Health Med., 15: 54-60.

3. Miyajima M, Arai H(2015): Evaluation of the Production and Absorption of Cerebrospinal Fluid. Neurol Med Chir (Tokyo), 55: 647-656.

4. Avery RA et al.(2010): Reference range for cerebrospinal fluid opening pressure in children. N Engl J Med., 363: 891-893.

5. Yamada $\mathbf{S}(\mathbf{2 0 1 4})$ : Cerebrospinal fluid physiology: visualization of cerebrospinal fluid dynamics using the magnetic resonance imaging Time-Spatial Inversion Pulse method. Croat Med J., 55: 337-346.

6. Akpede GO, Sykes RM, Abiodun PO(1992): Indications for lumbar puncture in children presenting with convulsions and fever of acute onset: experience in the Children's Emergency Room of the University of Benin Teaching Hospital, Nigeria. Ann Trop Paediatr., 12: 385-389.

7. Ward E, Gushurst CA(1992): Uses and technique of pediatric lumbar puncture. Am J Dis Child, 146: 11601165.

8. Doherty CM, Forbes RB(2014): Diagnostic Lumbar Puncture. Ulster Med J., 83: 93-102.

9. Engelborghs S et al.(2017): Consensus guidelines for lumbar puncture in patients with neurological diseases. Alzheimers Dement (Amst), 8: 111-126.

10. Wilson-Smith EM(2011): Procedural Pain Management in Neonates, Infants and Children. Rev Pain, 5: 4-12.

11. Ljungman G, Gordh T, Sorensen S, Kreuger A(2001): Lumbar puncture in pediatric oncology: conscious sedation vs. general anesthesia. Med Pediatr Oncol., 36: 372-379.

12. Lago $P$ et al.(2009): Guidelines for procedural pain in the newborn. Acta Paediatr., 98: 932-939.

13. Bruccoleri RE, Chen L(2011): Needle-entry angle for lumbar puncture in children as determined by using ultrasonography. Pediatrics, 127: e921-926.

14. Bilic E, Bilic E, Dadic M, Boban M(2003): Calculating lumbar puncture depth in children. Coll Antropol., 27: 623-626.
15. Chong SY, Chong LA, Ariffin H(2010): Accurate prediction of the needle depth required for successful lumbar puncture. Am J Emerg Med., 28: 603-606.

16. Coley BD, Shiels WE, 2nd, Hogan MJ(2001): Diagnostic and interventional ultrasonography in neonatal and infant lumbar puncture. Pediatr Radiol., 31: 399-402.

17. Chordas C(2001): Post-dural puncture headache and other complications after lumbar puncture. J Pediatr Oncol Nurs., 18: 244-259.

18. Lee LC, Sennett M, Erickson JM(2007): Prevention and management of post-lumbar puncture headache in pediatric oncology patients. J Pediatr Oncol Nurs., 24: 200-207.

19. Joffe $\mathbf{A R ( 2 0 0 7 ) : ~ L u m b a r ~ p u n c t u r e ~ a n d ~ b r a i n ~}$ herniation in acute bacterial meningitis: a review. J Intensive Care Med., 22: 194-207.

20. Mazor SS, McNulty JE, Roosevelt GE(2003): Interpretation of traumatic lumbar punctures: who can go home? Pediatrics, 111: 525-528.

21. Greenberg RG, Smith PB, Cotten CM, Moody MA, Clark RH, Benjamin DK, Jr.(2008): Traumatic lumbar punctures in neonates: test performance of the cerebrospinal fluid white blood cell count. Pediatr Infect Dis J., 27: 1047-1051.

22. Bonadio W(2014): Pediatric lumbar puncture and cerebrospinal fluid analysis. J Emerg Med., 46: 141-150.

23. Thomas V, Ahmed R, Qasim S(2008): Cerebro spinal fluid analysis in childhood bacterial meningitis. Oman Med J., 23: 32-33.

24. Burger B et al.(2003): Diagnostic cerebrospinal fluid examination in children with acute lymphoblastic leukemia: significance of low leukocyte counts with blasts or traumatic lumbar puncture. J Clin Oncol., 21: 184-188.

25. Hauser RG, Campbell SM, Brandt CA, Wang S(2017): Cost-Effectiveness Study of Criteria for Screening Cerebrospinal Fluid To Determine the Need for Herpes Simplex Virus PCR Testing. J Clin Microbiol., 55: 1566-1575.

26. Lopez Roa P, Alonso R, de Egea V, Usubillaga R, Munoz P, Bouza E(2013): PCR for detection of herpes simplex virus in cerebrospinal fluid: alternative acceptance criteria for diagnostic workup. J Clin Microbiol., 51: 2880-2883.

27. Vermeulen M, Hasan D, Blijenberg BG, Hijdra A, van Gijn J(1989): Xanthochromia after subarachnoid haemorrhage needs no revisitation. J Neurol Neurosurg Psychiatry, 52: 826-828.

28. Hurtt AE, Smith MO(1997): Effects of iatrogenic blood contamination on results of cerebrospinal fluid analysis in clinically normal dogs and dogs with neurologic disease. J Am Vet Med Assoc., 211: 866-867. 